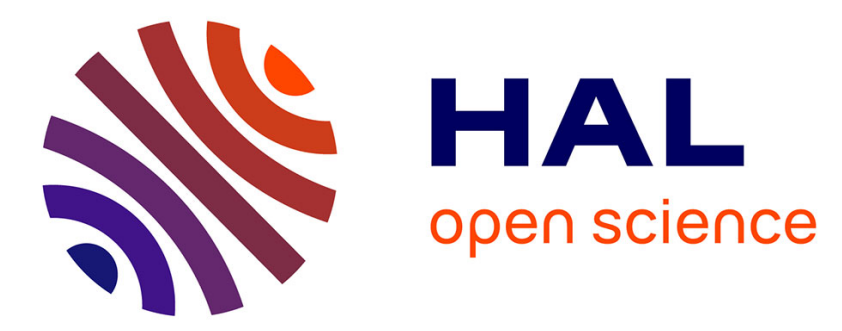

\title{
Force-Driven Polymerization and Turgor-Induced Wall Expansion
}

Olivier Ali, Jan Traas

\section{To cite this version:}

Olivier Ali, Jan Traas. Force-Driven Polymerization and Turgor-Induced Wall Expansion. Trends in Plant Science, 2016, 21 (5), pp.398 - 409. 10.1016/j.tplants.2016.01.019 . hal-01396586

\section{HAL Id: hal-01396586 https://hal.science/hal-01396586}

Submitted on 12 Oct 2018

HAL is a multi-disciplinary open access archive for the deposit and dissemination of scientific research documents, whether they are published or not. The documents may come from teaching and research institutions in France or abroad, or from public or private research centers.
L'archive ouverte pluridisciplinaire HAL, est destinée au dépôt et à la diffusion de documents scientifiques de niveau recherche, publiés ou non, émanant des établissements d'enseignement et de recherche français ou étrangers, des laboratoires publics ou privés. 


\section{Force-driven polymerization and turgor-induced wall}

\section{expansion}

4 Present address and affiliation:

5 1. Laboratoire de Reproduction et Développement des Plantes, Université Claude Bernard Lyon

6 1, ENS- Lyon, INRA, CNRS, Lyon, France

7 2. Virtual Plants Inria team, UMR AGAP, CIRAD, INRIA, INRA, Montpellier,

8 Corresponding Authors: Traas, J. (jan.traas@ens-lyon.fr), Ali, O. (olivier.ali@ens-lyon.fr) 


\section{Abstract}

10 While many molecular players involved in growth control have been identified in the past

11 decades, it is often unknown how they mechanistically act to induce specific shape changes

12 during development. Plant morphogenesis results from the turgor-induced yielding of the

13 extracellular, load-bearing cell wall. Its mechano-chemical equilibrium appears as a fundamental

14 link between molecular growth regulation and the effective shape evolution of the tissue. Here,

15 we focus on force-driven polymerization of the cell wall as a central process in growth control.

16 We propose that mechanical forces facilitate the insertion of wall components, in particular

17 pectins, a process that can be modulated through genetic regulation. We formalize this idea in a

18 mathematical model, which we subsequently challenge with published experimental results. 


\section{Introduction}

20 The link between gene regulation and growth, i.e. the irreversible spatial expansion of a cell or a

21 tissue, is a major issue in plant and animal developmental biology. However, although many

22 genetic regulators of morphogenesis have been identified, it is not clear how their activities

23 precisely translate into chemical and physical changes within the cells and consequently into

24 tissular morphogenesis. Here we will focus on the transduction between chemical and mechanical

25 energies, which is at the heart of this multi-scale process.

26 The particularities of plant growth and morphogenesis find their origin within the structure of

27 plant cells themselves, which can be considered as pressurized fluid droplets surrounded by a

28 stiff cell wall. In order to grow, cells must expand their wall in an irreversible manner. Early

29 work by e.g. Ray et al [1] provided evidence that wall expansion depends on chemical and

30 metabolic regulation, as opposed to a situation where turgor would simply induce growth by

31 breaking bonds between molecules. The current view is, that molecular networks that regulate

32 morphogenesis in plants have to interfere with the chemical structure of their wall, making it

33 yield to the internal turgor pressure at particular rates and in particular directions. To this end, the

34 role of transcriptional and post-transcriptional regulators in growth control can be understood in

35 terms of local wall remodeling.

36 From a thermodynamics perspective, plant morphogenesis can be seen as the result of an

37 interplay between turgor-generated mechanical energy and the chemical reactions leading to cell

38 wall expansion. Several conceptual frameworks have been developed to describe and analyze this

39 complex process ([2] for review, see also: [3], [4]). In this context, Barbacci et al exposed a

40 general thermodynamics framework to describe the coupling between mechanical and chemical 
41 energies [5]. In the present opinion paper we take this approach further by showing how a

42 specific biochemical and biomechanical process, force-driven polymerization, fits into this 43 framework.

44 Up to now, the coupling between mechanics and growth is usually considered from a large scale 45 perspective, individual walls being described as a continuous material, characterized by 46 rheological properties (see Glossary) such as elasticity or viscosity [6-8]. Hereby, growth 47 mechanisms are usually grasped through phenomenological equations similar to the ones 48 depicting plasticity in non-living materials. These continuum mechanics-based approaches are 49 very powerful as they benefit from existing simulation tools and concepts for the study of non50 biological materials. Despite the usefulness of such models, however, their large-scale focus is 51 also their limitation, for no attention is really paid to the underlying molecular mechanisms. As a 52 result, molecular regulations are abstractly interpreted as modulations of rheological variables.

53 Therefore it remains difficult to link experimentally measured transcriptional control or 54 enzymatic activity to observed growth patterns. Moreover, useful and intuitive interpretations of 55 underlying molecular functions such as repair mechanisms, self-assemblies, etc., are out of reach.

56 We therefore need, in addition, more mechanistic models for growth, including the detailed 57 molecular structure of the wall, explicitly linking molecular components to the overall 58 rheological properties.

59 We therefore investigated if, from a simplified description of the molecular content of the wall, 60 we could integrate a large-scale consecutive law of growing plant cells, which could then be used 61 to characterize the function of individual molecular regulators.

62 We argue here, that mechanical forces generated by turgor could facilitate the insertion of wall 
63 components. Such a coupling between mechanics and biochemistry, termed force-driven

64 polymerization, has also been described for the assembly of actin bundles in animal systems [9].

65 Starting from known descriptions of cell wall structure (see [10] and references therein) we

66 expose how force-driven polymerization could be central in scaling up molecular processes into

67 viscoplastic behavior and ultimately growth of multicellular structures.

\section{The cell wall is a fiber-reinforced hydrogel}

69 The cell wall is composed of a network of rigid cellulose microfibrils embedded in a matrix, 70 mostly composed of water, polysaccharides such as pectins and hemicelluloses, proteins and ions

71 [11]. This matrix corresponds to a biphasic mixture between a porous solid phase and a liquid 72 one [12]. Therefore, the cell wall as a whole can be considered as a fiber-reinforced hydrogel.

73 From a functional point of view, the wall performs two contrasting functions, as it has to resist 74 turgor-induced constraints whereas simultaneously it should be able to expand. Because of their 75 mechanical characteristics $[13,14]$, the cellulose microfibrils tethered to each other by shorter 76 hemicellulose chains are major actors in both of these functions (for reviews see: $[10,15][16]$.

77 The available experimental evidence suggests that cellulose microfibrils deposition is involved in 78 growth inhibition rather than in growth promotion per se. Indeed, after drug-induced inhibition of 79 cellulose deposition, cells can continue to grow during several tens of hours $[17,18]$. Similarly, 80 growth also occurs in certain mutant backgrounds where cellulose synthesis is impaired $[19,20]$.

81 These experimental clues suggest that growth of the cell wall relies on the expansion of the 82 embedding matrix. In other words a scenario has emerged, where cell wall expansion is restricted 83 by the synthesis of cellulose microfibrils and promoted by the synthesis of matrix material. In the 84 following paragraphs we will discuss an important component of this matrix, i.e. pectin. As we 
85 will see, there is increasing evidence that pectin could play an important role in growth control.

\section{Pectin is an important structural component of the wall matrix}

87 As indicated above, hemicelluloses such as xyloglucans have been classically considered as

88 essential structural components, central to concepts of wall loosening and induction of growth.

89 However, the recently produced Arabidopsis thaliana double mutant ( $x x t 1 / x x t 2)$, showing a loss

90 of xyloglucan synthesis, does not show major growth deficiencies $[21,22]$, suggesting that the

91 irreversible expansion of the cell wall does not rely, at least totally, on hemicellulose dynamics

92 only. This has revived the interest in the role of pectins in growth control. Pectins form up to 35

$93 \%$ of the primary cell wall of dicots [11] - i.e. the wall surrounding young and fast growing cells.

94 Their dynamics have been associated with growth control in several cell types, in particular tip-

95 growing cells such as pollen tubes and root hairs, for review see [10] and references therein, see

96 also e.g. [23], [24]. Moreover, AFM-based assays on Arabidopsis thaliana, demonstrated that

97 pectin demethyl-esterification was required and tightly regulated during cell wall softening prior

98 to aerial organ outgrowth [25], [26]. In addition, recent evidence indicates the existence of close

99 interactions between cellulose and pectins which could function as tethers between the

100 microfibrils $[15,27]$. Dwarf phenotypes in mutants where pectin synthesis or delivery is impaired

101 further illustrate the importance of pectin production for plant growth [28,29]. Noteworthy,

102 pectins have also been identified as important regulators of growth in more primitive plants such

103 as the green algae [30,31].

104 Pectins are produced in the Golgi and exocytosed within the cell wall. They are composed of four

105 different polysaccharides: Homogalacturonan, Rhamnogalacturonan I, Rhamnogalacturonan II

106 and Xylogalacturonan. Different pectin molecules correspond to various combinations of those 
107 three residues covalently attached together. Hereby, homogalacturonans stand out, for they 108 roughly account for $65 \%$ of all of them [32]. A fundamental feature of homogalacturonans is 109 their ability to form multiple intermolecular chelation bonds in presence of calcium cations.

110 These cations-dependent bonds are able to glue together pectin molecules, forming structures 111 called "egg-boxes" (Fig.1). In a cation-rich environment, these adhesive interactions lead to the 112 formation of a hydrogel [33]. In addition, pectins are able to interact with other cell wall 113 components including cellulose and hemicellulose [34].

114 An interesting feature is that newly synthesized homogalacturonans cannot spontaneously form 115 egg-box structures. Indeed, in order to trap divalent cations, the carboxyl groups of the 116 homogalacturonan chains must be negatively charged. When synthesized, carboxyl residues are 117 shielded by methyl groups, which must be removed to induce gelification. Therefore, two types 118 of pectin molecules can be distinguished: (i) "inactive" methyl-esterifed M-pectin and (ii) 119 "active", demethyl-esterified pectin, able to aggregate into macromolecular assemblies.

120 Demethyl-esterification is carried on within the cell wall by specialized enzymes, the pectin 121 methyl-esterases (PMEs). In turn, PME-inhibitors (PMEIs) can modulate PME activity. This 122 process is essential, for both the degree and the pattern of demethyl-esterification influence the 123 mechanical/rheological properties of the constituted gel [35]. Accordingly, modifications in the 124 levels of PME and PMEI expression significantly alter growth rates [36]. Note that other charged 125 residues such as acetyl-esters [11] can also be present on homogalacturonans and their regulation 126 could play a similar role. However, for the sake of simplicity we only consider methyl-ester 127 groups here. Noteworthy, 66 PMEs and 69 PMEIs have been reported in the Arabidopsis 128 genome, attesting for the importance of the process [33,37]. The PME/PMEI system appears as a 129 key regulator of cell wall expansion: 
- First, by regulating the amount of "active" demethyl-esterified pectins, within the matrix liquid phase, ready for insertion it influences its viscosity: The more active molecules available, the faster the expansion.

- Secondly, by removing more or less methyl groups on pectin molecules already stuck together, it regulates the strength of the bonds within the matrix solid phase. This can be interpreted as the tuning of the plastic yielding threshold of the cell wall.

136 In conclusion, in the previous paragraphs we have seen that the cell wall can be considered as a

137 fiber reinforced hydrogel, which is put under tension by the osmotic pressure within the cells. To

138 grow, the cells have to yield to this pressure by expanding their walls in an irreversible manner.

139 We will next discuss the chemical and physical processes that are at the basis of this expansion, 140 before indicating how molecular regulation can act to generate specific shapes of tissues and

141 organs. To be able to go beyond qualitative descriptions of growth regulation, we will present a

142 description in the form of a mathematical model. Finally we test this model using available 143 quantitative data

\section{Chemical equilibrium of the pectin hydrogel}

145 If single molecules in solution can bind to each other, macromolecular assemblies are generated.

146 Assuming a high enough initial concentration of those molecules, such a mechanism eventually

147 leads to the formation of a hydrogel, i.e. the percolation of a macromolecular solid phase within a

148 solution of single molecules. The pectin matrix corresponds to such a biphasic system [12]. When

149 the cell is growing, new matrix material has to be added: new components are exocytosed within

150 the liquid phase and components from the liquid phase have to be transferred into the solid one.

151 Because the wall is a closed shell of constant thickness (i.e. with no border where an hypothetic 
152 nucleation mechanism could take place), a way to add new components to the solid phase is by

153 inserting them in between existing ones. Such a mechanism is sketched in Fig.1a and formalized

154 in Eq.1, where $N_{b}$ and $M$ respectively depict one bond between two molecules in the solid phase

155 and a free molecule in the liquid one.

$$
N_{b}+M \rightleftarrows 2 N_{b}
$$

157 This chemical equilibrium between the liquid and solid phase of the gel can be formalized by 158 standard concepts from thermodynamics. Indeed, phase equilibrium is reached once the free 159 energy (we will drop the "free" for now on) difference $\Delta E\left(=E_{\text {bound }}-E_{\text {unbound }}\right)$ between the 160 two sides of Eq.1 vanishes. This energy difference reads $\Delta E=E_{b}-k_{B} T \cdot \ln \left(c / c_{0}\right)$ and 161 encompasses two general classic features. First, the stabilization of molecules by the adhesive 162 interactions between them is accounted for by the first term $E_{b}<0$ (the negative sign meaning 163 that adhesion stabilizes the system, i.e. lowers its energy). Second, the insertion of one molecule 164 from the liquid phase into the solid one comes with the loss of some degree of freedom. This is 165 expressed by the second term $-k_{B} T \cdot \ln \left(C / c_{0}\right)$ a standard expression of mixing entropy, where $166 k_{B} T$ represents the thermal energy of the system $\left(k_{B}\right.$ and $T$ respectively stand for the Boltzmann 167 constant and the absolute temperature), $c$ depicts the monomer concentration in the liquid phase 168 and $c_{0}$ the water concentration in that same phase $\left(\simeq 55 \mathrm{~mol} \cdot L^{-1}\right)$. In view of the above, the 169 equilibrium condition $\Delta E=0$ provides a value for a corresponding critical free molecule 170 concentration (with $\beta=1 / k_{B} T$ ):

$$
c_{0}^{*}=c_{0} \cdot \exp \left(\beta E_{b}\right)
$$

172 In other terms, the actual concentration $\mathrm{c}$ of monomers in solution controls the chemistry of the 
173 gel:

174

- If $c>c_{0}^{*} \Leftrightarrow \Delta E<0$, monomers spontaneously insert into the solid phase, growth

175 occurs.

176

- If $c<c_{0}^{*} \Leftrightarrow \Delta E>0$, no spontaneous insertion, no growth occurs.

177 The critical concentration $c_{0}^{*}$ only depends on one parameter: the adhesive energy between

178 molecules in the solid phase ( $E_{b}$ in Eq.2). In order to modulate this equilibrium the cell only has

179 to tune the adhesive links between molecules. This can be achieved using enzymatic activities,

180 i.e. through molecular regulation. However, another efficient way to do so is by pulling on those

181 links (Fig.1), as detailed in the following paragraph.

\section{Influence of mechanical forces on the chemical equilibrium}

183 A tensile force $(f)$ applied on the solid phase can ease the breaking of existing bonds, thus

184 facilitating the insertion of soluble molecules. This shifts the chemical equilibrium towards

185 polymerization - i.e. towards the right-hand side of Eq.1. In this case, the energy difference has

186 to be lowered by the work $w=f \cdot a$ generated by the tensile force $(f)$ over a distance $a$,

187 characteristic of the deformation of loaded bonds: $\Delta E^{\prime}=\Delta E-f \cdot a$. Consequently, the

188 equilibrium condition $\Delta E^{\prime}=0$ yields, this time, a force-dependent expression for the equilibrium

189 concentration $c^{*}$ :

$$
c^{*}(f)=c_{0}^{*} \cdot e^{-\beta \cdot f \cdot a}
$$

191 This mechanism assures that tensile forces applied on a gel at equilibrium decrease its critical 192 concentration. As a consequence molecules in solution are recruited into the solid phase, (Fig.1 \& 
193 2). This transduction mechanism is called force-induced polymerization and has been shown to

194 play an important role in the dynamics of major load-bearing structures such as the actin

195 cytoskeleton [38-41]. Note that this mechanism is by essence irreversible, for when an increased

196 force is applied on a given configuration (black arrow on Fig.2a) and then removed (dashed black

197 arrow on Fig.2a) the system does not go back to its original position.

198 Linking mechanics and biochemistry to geometry: spatial expansion

\section{9 of the pectin matrix under tensile force}

200 One difference between the growth of living tissues and the plasticity of non-living materials is

201 the mass increase of the former. Therefore, it seemed logical to seek for a growth law based on 202 the equation of mass conservation of the solid phase of the matrix (see Box 1 for details).

203 Formalizing the fact that the local expansion of the matrix is fuelled by the insertion mechanism 204 previously exposed yields (combination of Eq. 2, 3 \& B1-4):

$$
R_{g}=K \cdot \delta c \cdot e^{\beta\left(f \cdot a-E_{b}\right)}
$$

206 where the relative expansion rate $\left(R_{g}\right)$ is expressed as a function of the tensile force $(f)$, 207 formalizing the out-of-equilibrium insertion reaction. $K>0$ is a proportionality constant and $\delta c$ 208 represents the excess of free monomer in solution compared to the equilibrium concentration. 209 Note that in an exhaustive approach the coefficient $K$ should depend on the elastic energy stored 210 in the solid phase. But in a turgid growing tissue this stored energy should be roughly constant 211 and can be ignored for the sake of simplicity.

\section{Reinforcing the gel: the cellulose microfibrils network steps in}


213 Eq.4 depicts the expansion of a hydrogel under mechanical load, applied to the specific case of

214 pectin within the cell wall. A corollary question concerns the integration of the cellulose 215 microfibril network in this description.

216 The entanglement between the pectin-based matrix and the cellulose fibrils leads to a complex 217 distribution of mechanical constraints at the molecular scale. An accurate mechanical analysis 218 would require a precise structural description of the considered wall and intensive numerical 219 simulations, similar to the ones produced by Kha et al [42] and Puri et al [43] in the case of cell 220 wall models containing only cellulose and hemi-cellulose fibers. Such a study lies outside of the 221 range of this article and we will only provide a qualitative analysis here.

222 In a wall containing both pectin and cellulose, the force $(f)$ felt by a pectin strand in a given 223 direction should in principle only be a fraction of the total force $\left(f_{\text {tot }}\right)$ exerted on a unit volume of 224 the wall. This fraction would depend, at the very least, on the relative concentration of cellulose 225 fibers compared to pectin strands $\left(r_{c-p}\right)$ and the rigidity of those fibers $\left(Y_{c}\right)$ : $226 f=f\left(f_{\text {tot }}, r_{c-p}, Y_{c}\right) \leq f_{\text {tot }}$.

227 Controlling both the angular distribution of cellulose fibers and their rigidity appears as a way to 228 regulate the force exerted on pectin strands and consequently the expansion rate $\left(R_{g}\right)$ as 229 expressed in Eq.4. Following this idea, two stereotypical asymptotic cases can be evoked:

$230 \quad$ All the cellulose fibers are oriented perpendicular to the expanding pectin strand (c.f. Fig. 231 1b-2). In this case, the cellulose fibers and the pectin strand can be considered in series 232 and the whole force exerted on the wall is transmitted to the strand: $f=f_{\text {tot }}$.

233 - All the cellulose fibers are oriented in the same direction as the expanding pectin strand 
(c.f. Fig 1b-3). In this case, the cellulose fibers and the pectin strand lie in parallel and the $r_{c-p}$ and $r_{Y}$ respectively account for the ratio of relative concentrations of pectin and cellulose and the rigidity ratio between a pectin strand and a cellulose fiber.

238 The qualitative point we want to make here is that enhancing the deposition of cellulose in a 239 given direction should directly down-regulate the expansion rate of the matrix in this specific 240 direction.

241 Finally, it is worth mentioning that a detailed thermodynamical description of such a fiber242 reinforced hydrogel should not only include dissipation between matrix molecules but also 243 between the fibers themselves and between the fibers and the matrix.

\section{Experiments on Chara fit the force-driven polymerization model}

245 We were not aware of quantitative experimental studies on higher plants focused on the 246 relationship between molecular composition of the cell wall, turgor-generated forces and the 247 morpho-dynamics of the whole tissue. Fortunately, such studies have been conducted on the 248 green algae Chara corallina $[44,45]$ and we confronted the results with our model ( Fig.3).

249 In the case of isolated Chara cells, the mechanical force $(f)$ exerted on the cell wall is 250 proportional to the pressure differential $(\Delta P)$ between the inside and the outside of the wall.

251 Combined with Eq.4, this yields an exponential relationship between the pressure differential 252 exerted on a cell wall and its expansion rate. All other parameters kept constant, the ratio between 253 two relative growth rates $\left(R_{g} / R_{g}^{0}\right)$ as a function of the corresponding ratio of pressure 254 differentials $\left(\Delta P / \Delta P_{0}\right)$ reads: 


$$
\frac{R_{g}}{R_{g}^{0}}=\exp \left(\mathrm{C}_{1} \cdot \Delta P_{0} \cdot\left(\frac{\Delta P}{\Delta P_{0}}-1\right)\right)
$$

256 see Fig.3a for a comparison between experimental points and the fitting function.

257 If we assume that the insertion of demethyl-esterified pectins is the molecular mechanism behind 258 cell wall expansion, the growing rate is an affine function of the concentration of demethyl259 esterified pectin molecules in the solute phase. Fig.3b depicts this behavior and qualitatively 260 matches experimental evidence of this proportionality exposed on Fig.3 of [45]: putting Chara 261 cells in a pectin-enriched solution increased their growing response to mechanical stress.

262 Another qualitative feature of Chara growth observed by Proseus et al and reproduced by our 263 model is its dependency on temperature: Fig 3c depicts how the pressure-growth rate relationship 264 is affected by a slight decrease of temperature $(T)$, the shift from the plain curve to the dashed 265 one has to be compared to the shift between the two curves exposed on Fig.13 of [44]. These 266 qualitative agreements between our theoretical model and experimental data strengthen the idea 267 that force-driven assembly mechanisms of the cell wall matrix play an important role in cellular 268 growth.

\section{Discussion, perspectives \& conclusion}

270 Our ambition was to demonstrate that a fundamental molecular mechanism (force-driven 271 polymerization) applied to a key actor (pectin) can be at the heart of a complex integrated 272 behavior such as morphogenesis.

273 Although force-driven insertion appears as an appealing mechanism to explain cell wall 274 expansion at the molecular scale, it is worth noting that it might not be the only one possible. For 
275 instance, Rojas et al [23] proposed a detailed study of pollen tube expansion using an apposition

276 mechanism: Newly demethyl-esterified pectin molecules are not inserted into the solid phase but 277 rather directly polymerized as a new layer next to the old one. This new layer modifies the 278 mechanical properties of the load-bearing cell wall and induces its viscous relaxation. Such a 279 mechanism does not exclude the model we propose here but is rather complementary: Rojas et al 280 also describe a transduction mechanism between pectin chemistry and cell wall mechanics, 281 focusing their attention on the chemical control of the process. This shift of perspective between 282 their approach and ours (i.e. the question of causality between the chemical and the mechanical 283 states of the cell wall) is a molecular version of the debate between Linthillac and Schöpfer that 284 took place a few years back [46,47]: Is growth controlled by wall-loosening enzymes or by 285 turgor-induced stresses ? Our point is that what matters is the resulting equilibrium between both 286 ends which is formalized throught the critical concentration Eq.3.

287 Force-driven polymerization processes have been proposed to play a major part in several 288 biological functions relying on active gel dynamics and mechano-chemical transduction, from 289 actin filament formation and focal adhesion formation [9], [41], [48] (Kozlov et al) in animal 290 cells to cell wall instability and cell division in bacteria [49]. In our opinion, force-driven 291 polymerization processes could be as central in mechanobiology as Michaelis-Menten 292 mechanisms in biochemistry.

293 Indeed, force-driven polymerization can be seen as a transduction mechanism between a 294 mechanical force and a chemical reaction. From a theoretical perspective, in its full 3D version 295 this mechanism provides a gateway to develop models that integrate quantitative geometrical 296 variables and biochemical equations (Fig.4). In other words, it becomes possible to express 297 molecular activities in terms of geometrical outputs at the level of entire tissues and organs in a 
298 fully mechanistic manner. For this purpose, Eq.4 can be coupled to other differential equations 299 describing the dynamics of enzymatically-regulated variables. For instance one could assume that 300 the activation energy $E_{b}$ in Eq.4 depends on the demethyl-esterification degree of pectin 301 molecules and that pectin methyl esterases regulate its value. The influence of PME activity on 302 growth by coupling Eq.4 could then be explored using an equation describing PME dynamics.

303 To assess the validity of the model a wide range of experiments can be considered. The first step 304 would be to extend the quantitative analysis produced by Proseus and Boyer to cells and tissues 305 from higher plants: quantitative tracking to follow cells and tissues submitted to various levels of 306 turgor pressure could be a start. In complement, rescue assays could be tested to restore a normal 307 growth phenotype to mutant or drug-treated plants by modulation of mechanical forces. Finally 308 and more speculatively, the use and analysis of biomimetic systems such as artificial walls could 309 also be very useful. 


\section{Bibliography}

3111 Ray, P.M. and Ruesink, A.W. (1962) Kinetic experiments on the nature of the growth

312 mechanism in oat coleoptile cells. Dev. Biol. 4, 377-397

3132 Ali, O. et al. (2014) Physical models of plant development. Annu. Rev. Cell Dev. Biol. 30,

$314 \quad 59-78$

3153 Kennaway, R. et al. (2011) Generation of Diverse Biological Forms through Combinatorial

316 Interactions between Tissue Polarity and Growth. PLoS Comput Biol 7, e1002071-22

3174 Hamant, O. et al. (2008) Developmental Patterning by Mechanical Signals in Arabidopsis.

$318 \quad$ Science 322, 1650-1655

3195 Barbacci, A. et al. (2013) Another Brick in the Cell Wall: Biosynthesis Dependent Growth

$320 \quad$ Model. PLoS ONE

3216 Boudon, F. et al. (2015) A computational framework for 3D mechanical modeling of plant

322

323

324

325

326

327

328

329

330

331

332

333

334

335

336

337

338

339

340

341

342

343

344

345

346

347

348

349

350

351

352

353 morphogenesis with cellular resolution. PLoS Comput Biol 11, e1003950

7 Dyson, R.J. and Jensen, O.E. (2010) A fibre-reinforced fluid model of anisotropic plant cell growth. J. Fluid Mech. 655, 472-503

8 Dumais, J. et al. (2006) An anisotropic-viscoplastic model of plant cell morphogenesis by tip growth. Int. J. Dev. Biol. 50, 209-222

9 K Kruse, J.F.J. (2004) Aster, Vortices and Rotating Spirals in Active Gels of Polar Filaments. Phys. Rev. Lett. 92, 1-4

10 Wolf, S. et al. (2012) Growth Control and Cell Wall Signaling in Plants. Annu. Rev. Plant Biol. 63, 381-407

11 Caffall, K.H. and Mohnen, D. (2009) The structure, function, and biosynthesis of plant cell wall pectic polysaccharides. Carbohydrate Research 344, 1879-1900

12 Iijima, M. et al. (2005) Swelling behaviour of calcium pectin hydrogels by thermomechanical analysis in water. Thermochimica Acta 431, 68-72

13 Vincent, J. (1999) From cellulose to cell. Journal of Experimental Biology 202, 3263-3268

14 Zhang, T. et al. (2013) Visualization of the nanoscale pattern of recently-deposited cellulose microfibrils and matrix materials in never-dried primary walls of the onion epidermis. Cellulose 21(2), 853-862

15 Cosgrove, D.J. (2014) Re-constructing our models of cellulose and primary cell wall assembly. Current Opinion in Plant Biology 22, 122-131

16 Cosgrove, D.J. (1993) How do plant cell walls extend? Plant Physiology 102, 1-6

17 Heisler, M.G. et al. (2010) Alignment between PIN1 Polarity and Microtubule Orientation in the Shoot Apical Meristem Reveals a Tight Coupling between Morphogenesis and Auxin Transport. PLoS Biol. 8, e1000516

18 Pelletier, S. et al. (2010) A role for pectin de-methylesterification in a developmentally regulated growth acceleration in dark-grown Arabidopsis hypocotyls. New Phytologist 188, 726-739

19 Fagard, M. et al. (2000) PROCUSTE1 Encodes a Cellulose Synthase Required for Normal Cell Elongation Specifically in Roots and Dark-Grown Hypocotyls of Arabidopsis. The Plant Cell 12, 2409-2423

20 Hématy, K. et al. (2007) A receptor-like kinase mediates the response of Arabidopsis cells to the inhibition of cellulose synthesis. Current Biology 17, 922-931

21 Park, Y.B. and Cosgrove, D.J. (2012) A revised architecture of primary cell walls based on 
biomechanical changes induced by substrate-specific endoglucanases. Plant Physiology 158, 1933-1943

22 Cavalier, D.M. et al. (2008) Disrupting two Arabidopsis thaliana xylosyltransferase genes results in plants deficient in xyloglucan, a major primary cell wall component. The Plant Cell Online 20, 1519-1537

23 Rojas, E.R. et al. (2011) Chemically Mediated Mechanical Expansion of the Pollen Tube Cell Wall. Biophysical Journal 101, 1844-1853

24 Parre, E. and Geitmann, A. (2005) Pectin and the role of the physical properties of the cell wall in pollen tube growth of Solanum chacoense. Planta 220,582-592

25 Braybrook, S.A. and Peaucelle, A. (2013) Mechano-Chemical Aspects of Organ Formation in Arabidopsis thaliana: The Relationship between Auxin and Pectin. PLoS ONE 8, e57813

26 Peaucelle, A. et al. (2015) The Control of Growth Symmetry Breaking in the Arabidopsis Hypocotyl. Current Biology 25, 1746-1752

27 Wang, T. et al. (2015) Cellulose-Pectin Spatial Contacts Are Inherent to Never-Dried Arabidopsis Primary Cell Walls: Evidence from Solid-State Nuclear Magnetic Resonance. Plant Physiology 168, 871-884

28 Bouton, S. et al. (2002) QUASIMODO1 encodes a putative membrane-bound glycosyltransferase required for normal pectin synthesis and cell adhesion in Arabidopsis. The Plant Cell Online 14, 2577-2590

29 Gendre, D. et al. (2013) Trans-Golgi network localized ECHIDNA/Ypt interacting protein complex is required for the secretion of cell wall polysaccharides in Arabidopsis. The Plant Cell 25, 2633-2646

30 Proseus, T.E. and Boyer, J.S. (2006) Calcium pectate chemistry controls growth rate of Chara corallina. J. Exp. Bot. 57, 3989-4002

31 Proseus, T.E. and Boyer, J.S. (2014) Pectate chemistry links cell expansion to wall deposition in Chara corallina. psb 7, 1490-1492

32 Mohnen, D. and Bar-Peled, M. (2008) Cell wall polysaccharide synthesis. ... the Plant Cell Wall for ...

33 Wolf, S. et al. (2009) Homogalacturonan Methyl-Esterification and Plant Development. Mol. Plant 2(5), pp.851-860

34 Popper, Z.A. and Fry, S.C. (2008) Xyloglucan-pectin linkages are formed intraprotoplasmically, contribute to wall-assembly, and remain stable in the cell wall. Planta 227, 781-794

35 Peaucelle, A. et al. (2011) Pectin-Induced Changes in Cell Wall Mechanics Underlie Organ Initiation in Arabidopsis. Current Biology 21, 1720-1726

36 Peaucelle, A. et al. (2008) Arabidopsis phyllotaxis is controlled by the methyl-esterification status of cell-wall pectins. Curr. Biol. 18, 1943-1948

37 Sénéchal, F. et al. (2014) Homogalacturonan-modifying enzymes: structure, expression, and roles in plants. J. Exp. Bot. 65, 5125-5160

38 Kozlov, M.M. and Bershadsky, A.D. (2004) Processive capping by formin suggests a forcedriven mechanism of actin polymerization. The Journal of Cell Biology 167, 1011-1017

39 Courtemanche, N. et al. (2013) Tension modulates actin filament polymerization mediated by formin and profilin. Proc Natl Acad Sci USA 110, 9752-9757

40 Shemesh, T. and Kozlov, M.M. (2007) Actin Polymerization upon Processive Capping by Formin: A Model for Slowing and Acceleration. Biophysical Journal 92, 1512-1521

41 Shemesh, T. (2005) Focal adhesions as mechanosensors: A physical mechanism. 
400

401

402

403

404

405

406

407

408

409

410

411

412

413

414

415

416

417

418

419
Proceedings of the National Academy of Sciences 102, 12383-12388

42 Kha, H. et al. (2010) WallGen, Software to Construct Layered Cellulose-Hemicellulose Networks and Predict Their Small Deformation Mechanics. Plant Physiology 152, 774-786

43 Yi, H. and Puri, V.M. (2012) Architecture-Based Multiscale Computational Modeling of Plant Cell Wall Mechanics to Examine the Hydrogen-Bonding Hypothesis of the Cell Wall Network Structure Model. Plant Physiology 160, 1281-1292

44 Proseus, T.E. et al. (2000) Turgor, temperature and the growth of plant cells: using Chara corallina as a model system. J. Exp. Bot. 51, 1481-1494

45 Proseus, T.E. and Boyer, J.S. (2007) Tension required for pectate chemistry to control growth in Chara corallina. J. Exp. Bot. 58, 4283-4292

46 Wei, C. and Lintilhac, P.M. (2003) Loss of stability - a new model for stress relaxation in plant cell walls. Journal of theoretical biology 224, 305-312

47 SCHOPFER, P. (2008) Is the loss of stability theory a realistic concept for stress relaxationmediated cell wall expansion during plant growth? Plant Physiology 147(3), 935-936 author reply $936-938$

48 Nicolas, A. et al. (2004) Cell mechanosensitivity controls the anisotropy of focal adhesions. Proceedings of the National Academy of Sciences 101, 12520-12525

49 Jiang, H. et al. (2011) Mechanical Control of Bacterial Cell Shape. Biophysj 101, 327-335 


\section{Boxes}

\section{Trends box:}

422 - Cellulose microfibrils have historically drained most of the attention of biomechanical 423 studies of the cell wall. Recent results, however, suggest that the soft pectin matrix in 424 which they are embedded might also play a significant role in the physico-chemical 425 equilibrium of growing cells.

426 - At the molecular scale, biophysicists have shown how mechanical forces applied on 427 molecular assemblies can modulate their chemical state and therefore initiate specific 428 biological responses.

429 - At the tissular scale, numerical simulation tools borrowed from material sciences are 430 increasingly used in developmental biology. Morphogenesis in plant tissues is particularly 431 suited for this kind of approach.

432 - Bridging the gap between the molecular and the tissular scales is a major challenge in 433 developmental biology, for it would help relating explicitly shape changes and specific 434 molecular actors. 


\section{Glossary box:}

436 Rheology: Study of the deformation of matter at a mesoscopic or macroscopic scale.

437 Viscosity: Rheological/mechanical property that relates the deformation rate of a material to its

438 mechanical load. For a given loading force, the more viscous a material is the slower it will

439 deform. Note that its inverse is called extensibility and is often used in growth modeling.

440 Thermal energy: The portion of the energy of a system due to the random relative microscopic

441 movements of its components. Absolute temperature of the system is, by definition, a direct

442 measure of this internal energy. For living systems which temperature is around $300 \mathrm{~K}$ the usual

443 value of their thermal energy is: $\mathcal{E}_{t h} \propto k_{B} T=1 / \beta \approx 4 \cdot 10^{-21} \mathrm{~J}$, where $k_{B}=1.38 \cdot 10^{-23} \mathrm{~J}$.

$444 K^{-1}$ and $T=300 \mathrm{~K}$ respectively stand for the Boltzmann constant and the absolute temperature.

445 Work of a force: Considering an object in motion and summited to a force, the work developed

446 by this force corresponds to the scalar product between the force vector and the displacement 447 vector. 


\section{Box 1: From matter conservation to force-induced expansion}

449 The conservation equation of the number of bonds in the solid phase reads:

$$
\frac{d n_{b}}{d t}=-n_{b} R_{g}+s_{b}
$$

451 The term of the left hand-side of Eq.B1-1 depicts the local time evolution of the bond 452 density (noted $n_{b}$ ). The first term of the right hand-side is proportional to the relative 453 expansion rate of the cell wall $\left(R_{g}\right)$ and accounts for the fact that the matrix can expand.

454 The minus sign in front of it insures that spatial expansion of the matrix $\left(R_{g}>0\right)$ tends to 455 diminish the local bond density. Finally, the second term of the right hand-side (named 456 the source term and denoted $s_{b}$ ) grasps the chemical insertion mechanism that transfers 457 molecules from the liquid phase into the solid one when the two phases are out of 458 equilibrium.

459 Because the cell wall is constantly under tension, the spacing between molecules in the 460 solid phase and therefore the bond density can be assumed constant over time (i.e. $461 d n_{b} / d t=0$ in Eq.B1-1). This assumption implies that the two terms of the right hand462 side of Eq.B1-1 compensate each other, leading to a direct relationship between the 463 expansion rate of the cell wall and the pectin chemical insertion rate:

$$
n_{b} R_{g}=s_{b}
$$

465 The proper derivation of chemical insertion rate $\left(s_{b}\right)$ from thermodynamical 
466 considerations lies outside the range of this opinion article but in a few words it can be

467 estimated as follows. It should be proportional to the density of insertion sites $\left(n_{b}\right)$ and to 468 the excess of free molecules in solution compared to equilibrium: $\delta c=c-c^{*}$. It 469 therefore corresponds to a relaxation term when the pectin molecules in the two phases 470 are out of equilibrium. If the system is only slightly out of equilibrium, this relaxation 471 flux can be linearized with respect to the chemical potential difference between the two 472 pectin phases $\left(\partial \Delta \varepsilon /\left.\partial c\right|_{c^{*}}\right.$ in Eq.B1-3) leading to the following expression:

473

$$
s_{b} \propto-\left.\frac{\partial \Delta \varepsilon}{\partial c}\right|_{c^{*}} \cdot \delta c \cdot n_{b} \quad(\mathrm{~B} 1-3)
$$

474 This yields the simple relationship between the relative spatial expansion rate of the wall 475 and the equilibrium concentration $c^{*}(f)$ that explicitly depends on the mechanical force $f$ 476 as Eq.3 depicts:

$$
R_{g} \propto \frac{1}{c^{*}(f)}(\mathrm{B} 1-4)
$$




\section{Figures legends}

\section{$479 \quad$ Figure 1: Pectin strand dynamics \& mechanical load distribution.}

480 (a) Details on the matrix expansion mechanism. (a-1): demethyl-esterified pectin chain at 481 rest in equilibrium with DM pectin molecules in solution (light blue). (a-2): When loading 482 with a mild force the molecules constituting the chain are elastically deformed. Egg-box 483 structures are undeformed and the corresponding bond strong. (a-3): If the loading force is 484 high enough (above a threshold value defined as $f_{t h}=E_{b} / a$ ), egg-box structures are 485 mechanically deformed, their corresponding strength is lowered. Consequently, they can be 486 destabilized by close-by, demethyl-esterified pectin molecules in solution. (a-4): Once the 487 initial bond broken, the destabilizing molecule is inserted, increasing the total length of the 488 chain. (b) Influence of of cellulose microfibrils orientation. (b-1): Structural sketch of a 489 fiber reinforced hydrogel. The big purple bars represent cellulose microfibrils, the smaller 490 orange one pectin strand composing the solid phase and the blue dots stand for the free pectin 491 molecules in the liquid phase. We assume that cellulose fibrils are deposited by a preferred 492 direction. Within this 2D representation, two 1D cases are evoked: (b-2): The studied pectin 493 strand is perpendicular to the cellulose microfibrils main direction. In that case, the two 494 structure can be assume in series and the forces they experience are the same: $f_{\text {tot } y}=f_{c y}=$ $495 \quad f_{y}$ where those forces respectively correspond to the total force felt by the wall, the fraction 496 felt by the cellulose fiber and the fraction felt by the pectin strand. (b-3): The studied pectin 497 strand is parallel to the main cellulose microfibrils direction. The total force exerted on the 498 wall is distributed between both structures, this time in parallel: $f_{\text {tot } x}=f_{c x}+f_{x}$; the 
499 fraction felt by the pectin strand is consequently lower than the total force: $f_{x} \leq f_{\text {tot } x}$. 


\section{Figure 2: Phase diagram of an hydrogel under tensile force.}

501 The abscise axis quantifies the work developed by the tensile force $(w=f \cdot a)$ compared to the

502 thermal energy available $\left(\beta=1 / k_{B} T\right)$. The ordinate axis shows the normalize concentration of

503 free active pectin molecules in the liquid phase. The orange curve is a visual representation of

504 Eq.3 and therefore represents the chemical equilibrium between the solid and the liquid phase of

505 the gel. Each point of the plane represents one configuration of the gel that corresponds to a

506 specific couple "concentration / force", the points under the equilibrium curve correspond to gels

507 that do not expand their solid phase whereas points above do. Sub-figures (a) \& (b) show two

508 simple putative growth scenarios: (a) From an initial non-growing state (black dot) we assume an

509 increase of the mechanical load, that translates onto the phase plan as a rightward shift of the

510 point describing the system state (gray dot). Being this time in the "growth zone", spontaneous

511 insertion of monomers into the solid phase spontaneously happens. Note that from the initial

512 black point it takes a minimal force (dashed part of the arrow) to get into the "growth zone", this

513 can be interpreted as a force threshold $\mathrm{fth}$, depicted by the vertical dotted orange line. Finally the

514 gray dashed arrow symbolizes the "out of equilibrium" driving force of the polymerization

515 process that tends to diminish the concentration of free molecules in solution. Note that if the

516 mechanical force goes back to its initial value (plain gray arrow), the system does not recover its

517 initial state (black dot) but a new one (orange dot). (b) This time growth is initiated by a release

518 of demethyl-esterified pectin in the liquid phase, again a threshold phenomenon is observed, this

519 time in terms of concentration $c_{t h}$, depicted by the horizontal dotted orange line. 


\section{Figure 3: Growth rate dependency on demethyl-esterified pectin}

\section{1 concentration, turgor pressure and temperature; comparison}

\section{2 between experimental data and model.}

523 (a): Influence of turgor pressure on relative expansion rate. Comparison between

524 experimental data and Eq.4. Dots represent experimental published data from (Proseus et al.

525 2000) and error bars the precision of our reading of these data. The dashed curve represents the

526 best fit of these points by the exponential function exposed in Eq. 5: $R_{g} / R_{g}^{0}=\exp \left(C_{1} \Delta P 0\right.$.

$\left.527\left(\Delta P / \Delta P_{0}-1\right)\right)$ where $C_{1}$ is the fitting coefficient. $\Delta P_{0}$ and $R_{g}^{0}=R_{g}\left(\Delta P_{0}\right)$ are arbitrary values

528 taken as references. (b)\&(c): Qualitative influence of various parameters on the growth behavior.

529 (b): Influence of an increase of free demethyl-esterified pectin concentration in the liquid

530 phase ( $\boldsymbol{\delta} \boldsymbol{c}$ in Eq.4). As on sub-figure (a), the curves depict the evolution of a normalized growth

531 rate $R_{g} / R_{g}^{0}$ with respect to a normalized pressure differential $\left(\Delta P / \Delta P_{0}\right)$ and mimick Fig.3B

532 in[45]. The plain curve corresponds to the best-fit curve exposed on sub-figure (a). The dashed

533 curve is deduced from the plain one by a two-fold increase of the free demethyl-esterified pectin

534 concentration $(\delta c)$. (c): Influence of the temperature. This time the temperature dependency

535 exposed in Eq.4 is investigated. $P_{0}, T_{0}$ and $R_{g}^{0}=R_{g}\left(\Delta P_{0}, T_{0}\right)$ are still arbitrary reference values.

536 The plain curve corresponds to the best-fit curve exposed on sub-figure (a). The dashed curve is

537 deduced from the plain one by using a smaller value of the temperature similar to the drop of

538 temperature studied by Proseus et al. in [44], see Fig.13-B. 


\section{Figure 4: Transducing chemical \& mechanical inputs into}

\section{0 morphological outputs}

541 The morphological evolution of the tissue is quantified by its relative growth rate $R_{g}$. The growth

542 law that relates it to turgor-induced mechanical forces and to the cell wall mechano-chemical

543 properties is a direct consequence of the cell wall molecular organization. Its expression exposed

544 here corresponds to the combination of Eqs.4 \& 5. From a functional perspective the growth law

545 can be seen as the final step of the integrative chain between gene expression and shape

546 evolution. It also appears as the step where biochemical properties (quantified by scalar variables,

547 i.e. « simple» numbers) are combined with mechanical and geometrical properties (quantified by

548 oriented variables, namely vectors and matrices). 
(A-1) $l_{0}$
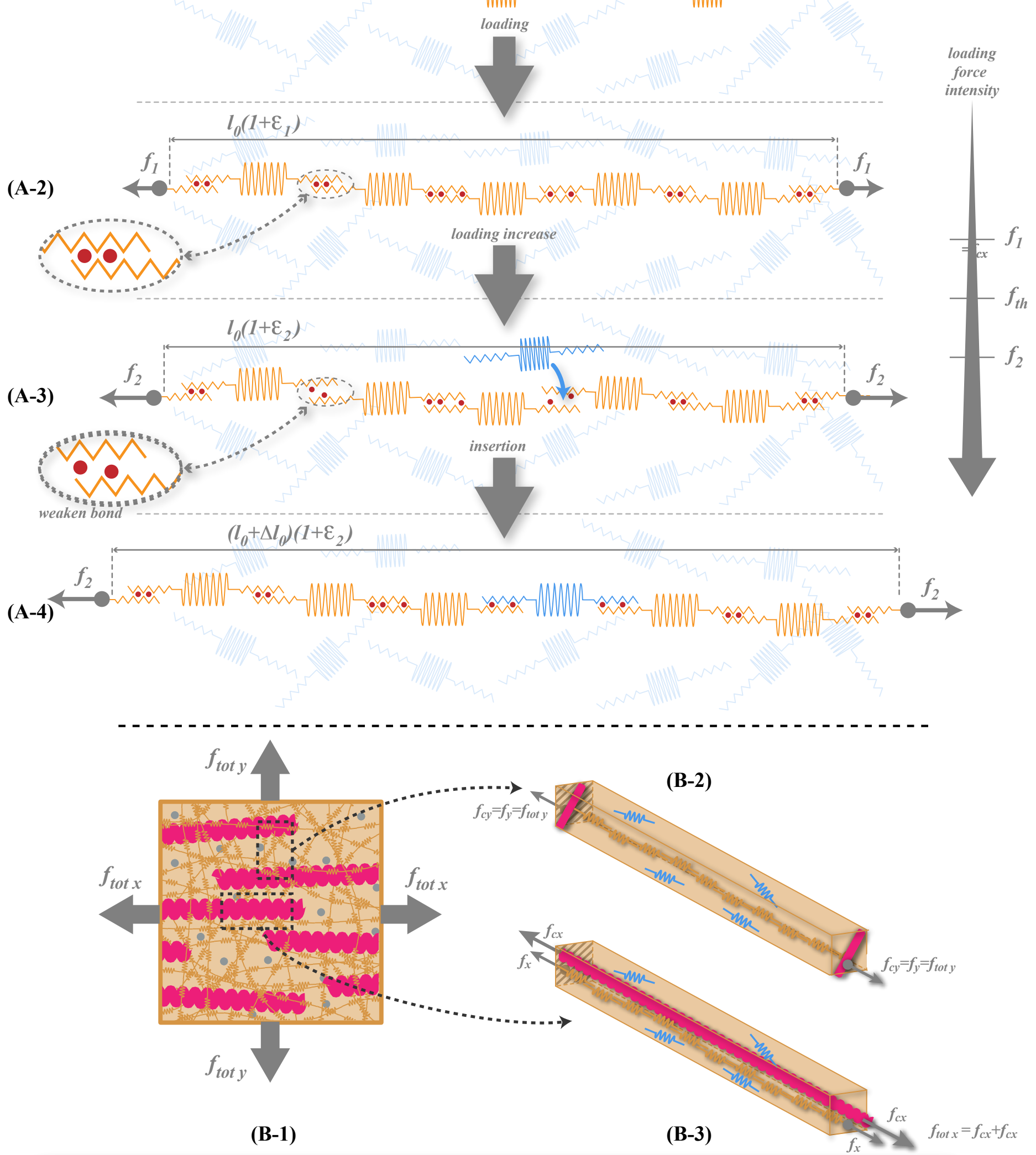

Loading force on a matrix strand


(A)

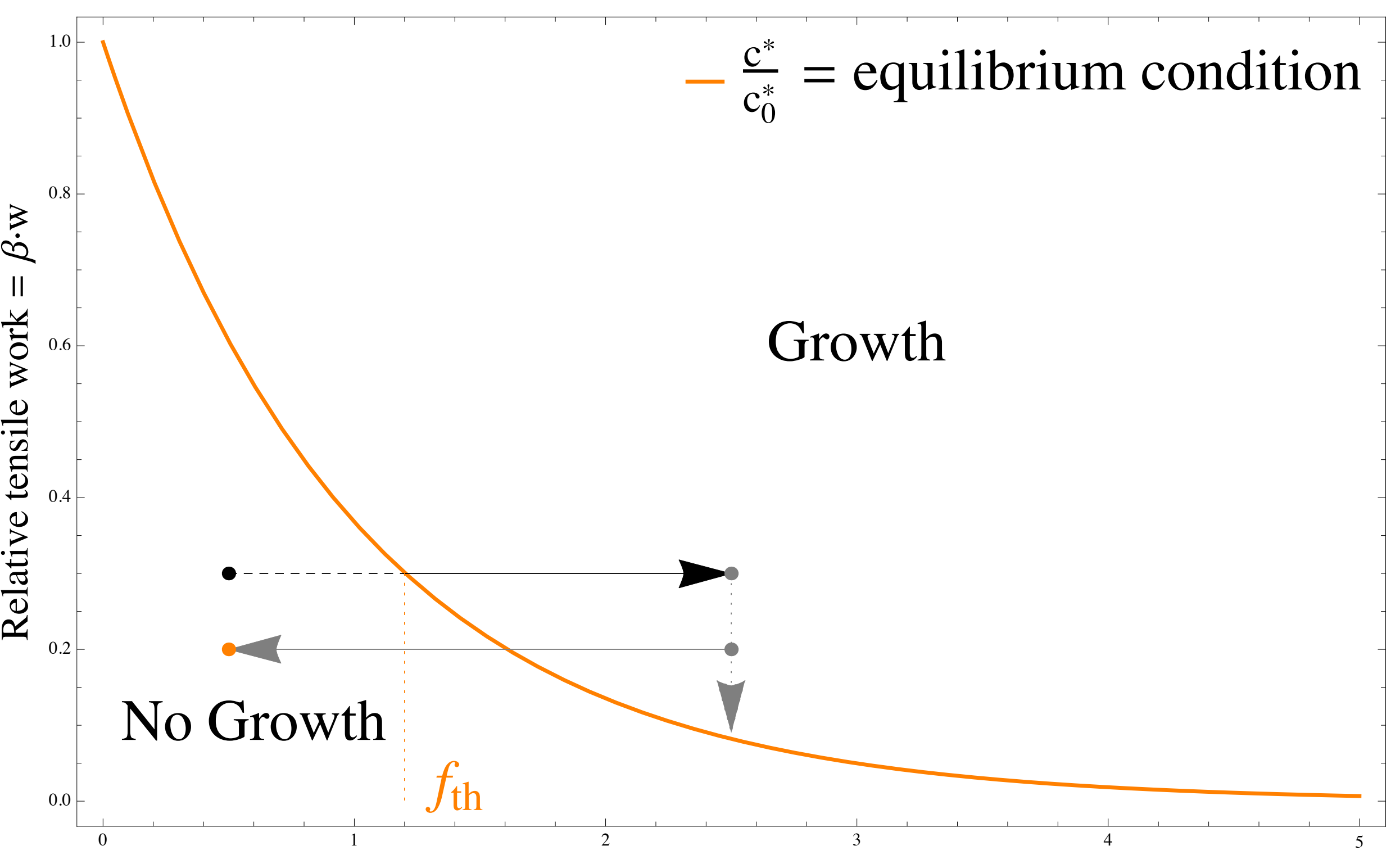

Relative free monomer concentration $=\mathrm{c} / c_{0}^{*}$
(B)

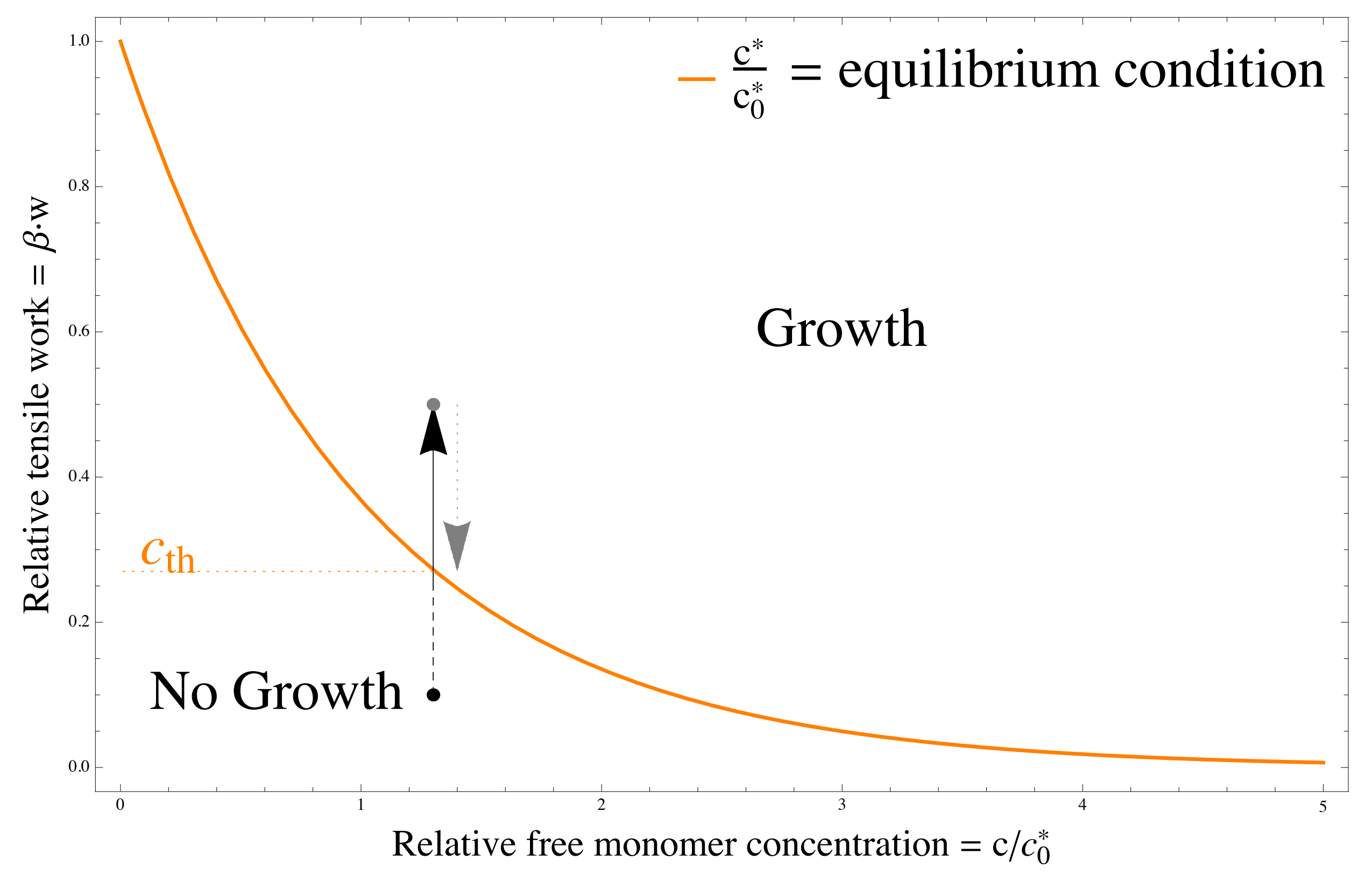


(A)

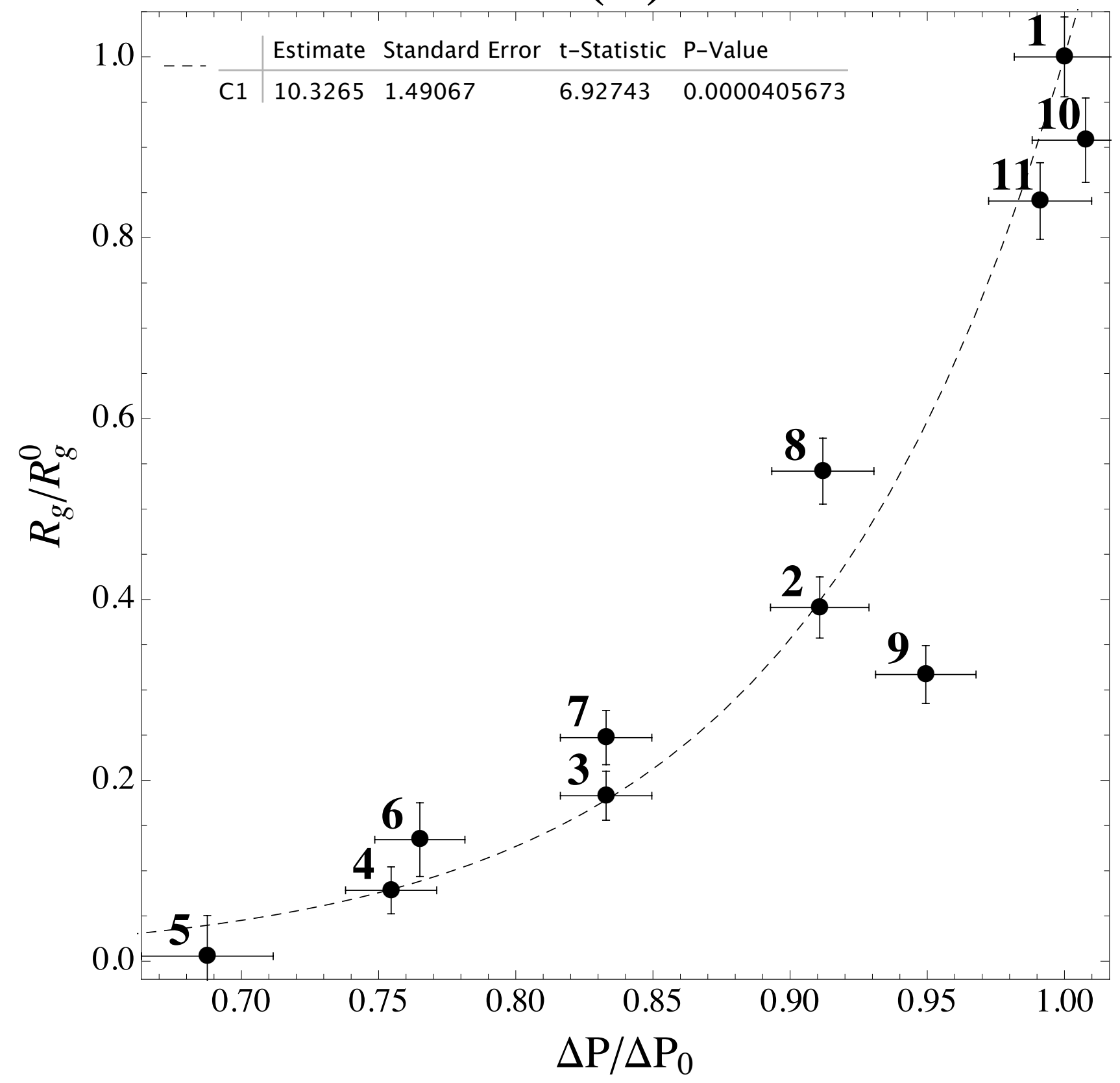

(B)
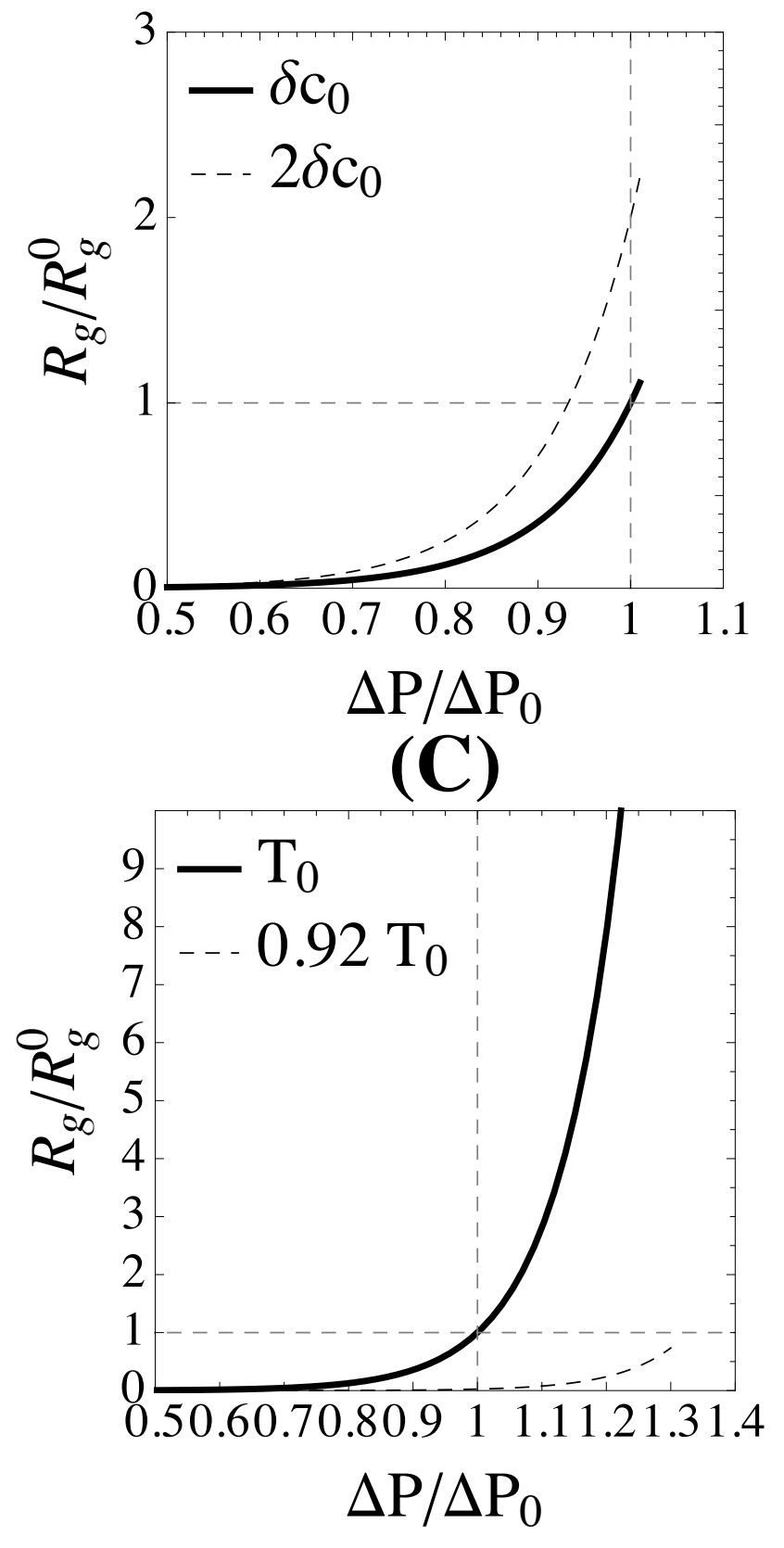


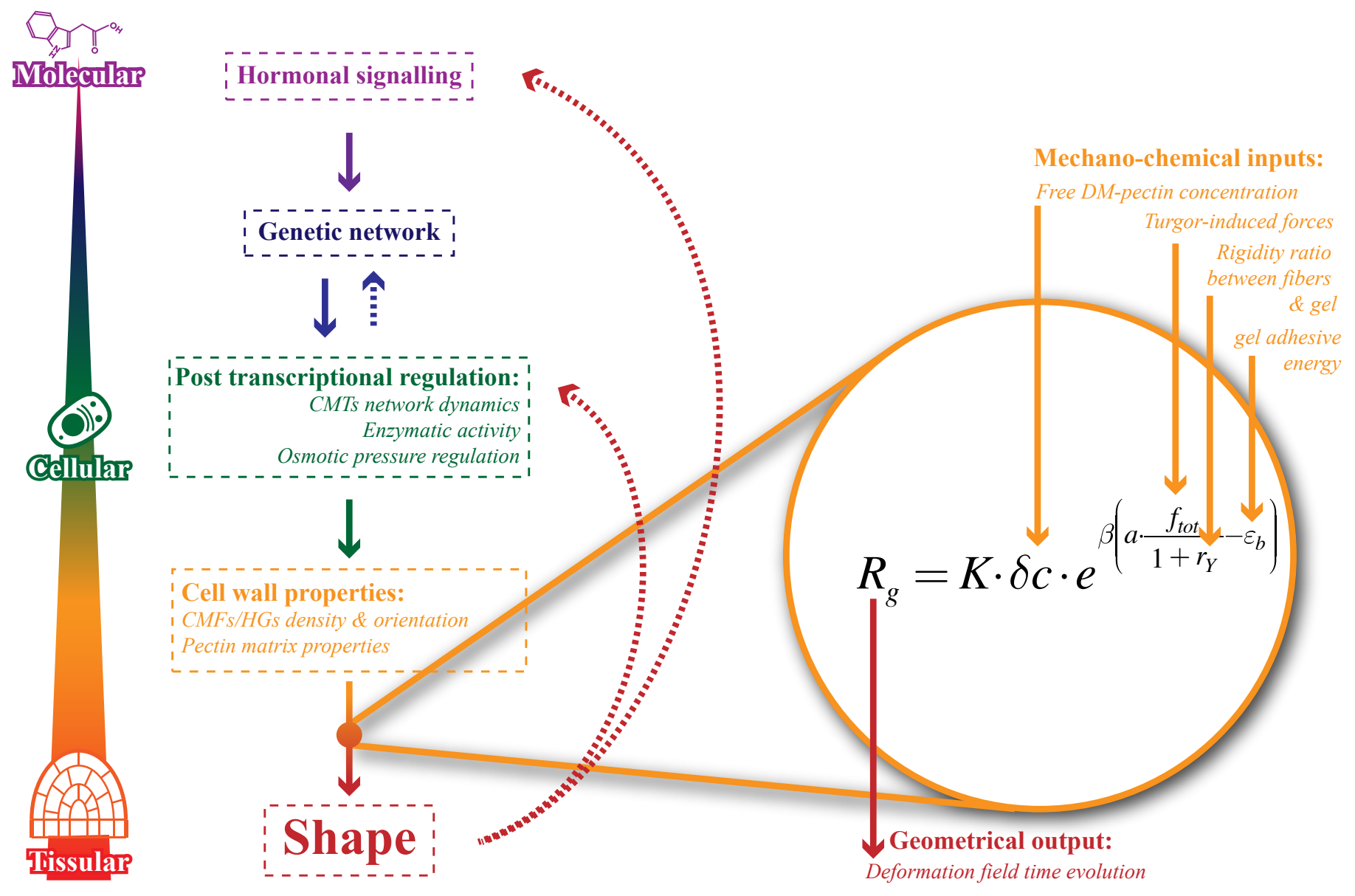

